\title{
IS THE PRODUCT OF CCC SPACES
}

\section{A CCC SPACE?}

\author{
NINA M. ROY \\ This paper is dedicated to John C. Oxtoby, who sparked the author's \\ interest in the subject.
}

\section{Introduction}

A ccc space is a topological space which satisfies the countable chain condition: Each family of (pairwise) disjoint nonempty open sets is countable. A separable space, for instance, is a ccc space. This is so because given a family $\mathcal{F}$ of disjoint nonempty open sets in a separable space $X$, one can define an injection of $\mathcal{F}$ into a countable dense subset $S$ of $X$ by choosing one point of $S$ in each member of $\mathcal{F}$. A ccc space, however, need not be separable. For example, if $I$ is a set with cardinality greater than $|\mathbb{R}|$ (the cardinality of the set of real numbers) and for each $i \in I, X_{i}=\{0,1\}$ with the discrete topology, then the product space $\Pi_{i \in I} X_{i}$ is $c c c$ but not separable [10, p. 51]. A simple example of a topological space which is not ccc is any uncountable set with the discrete topology. In a 1947 paper [12], E. Marczewski gave a proof (which may also be found in [15]) of Pondiczery's theorem that the product of at most $\mid \mathbf{R}$ | separable spaces is separable; and he raised the question as to whether the product of (just) two $c c c$ spaces is a $c c c$ space. It turns out that special axioms are needed to answer this question: it has a negative answer in the presence of the Continuum Hypothesis $(\mathrm{CH})$ and an affirmative answer when Martin's Axion and the negation of $C H$ are assumed. The main goal of this paper is to give fairly self-contained proofs of these two assertions, which we do in Sections 
2 and 3. Related topics (Souslin's Hypothesis, Property (K)) are touched upon in Sections 4 and 5 . None of the results and proofs are new.

First, some preliminaries. Let us recall that an ordinal number may be identified with the set of all smaller ordinal numbers; thus for ordinals $\alpha$ and $\beta$, the statements $\alpha<\beta, \alpha \epsilon \beta$, and $\alpha \subset \beta$ are all equivalent. The smallest infinite ordinal and the smallest uncountable ordinal will be denoted by $\omega$ and $\omega_{1}$ respectively. We will need the fact that $\omega$ and $\omega_{1}$ are also cardinal numbers. (A good reference for cardinal and ordinal numbers is [6].) The cardinality of the set of all functions from $\omega$ to 2 is $2^{\omega}$, and $2^{\omega}=|\mathbf{R}|$. The Continutum Hypothesis $(\mathrm{CH})$ is the statement $\omega_{1}=2^{\omega}$. The negation of $C H$, denoted here by $\neg C H$, is therefore the statement $\omega_{1}<2^{\omega}$. Gödel and Cohen proved that $C H$ is independent of the usual axioms of set theory, namely, the Zermelo-Fraenkel axioms together with the Axiom of Choice (usually denoted by $Z F C$ ).

We review the definition of the Gleason space of a topological space $X$, for use several times in the sequel. An open subset $U$ of $X$ is called a regular open set if $U$ is equal to the interior of its closure, i.e., $U=(\bar{U})^{0}$. The set $\mathcal{R}(X)$ of all regular open subsets of $X$ forms a complete Boolean algebra with respect to the operations $U \wedge V=U \cap V, U \vee V=(\overline{U \cup V})^{0}$, and $U^{\prime}=(X \backslash U)^{0}$. The Gleason space of $X$, denoted here by $G(X)$, is the Stone space of $\mathcal{R}(X)$. The space $G(X)$ is constructed as follows. A subset $\mathcal{F}$ of $\mathcal{R}(X)$ is called a filter of $\mathcal{R}(X)$ if (i) $\phi \notin \mathcal{F}$, (ii) $U \cap V \in \mathcal{F}$ for all $U, V \in \mathcal{F}$, and (iii) $V \in \mathcal{F}$ if $U \subset V$ and $U \epsilon \mathcal{F}$. An ultrafilter of $\mathcal{R}(X)$ is a filter which is not properiy contained in any filter of $\mathcal{R}(X)$. Then $G(X)$ is the set of all ultrafilters of $R(X)$. For each open set $U$ in $X$, let

$$
U^{*}=\left\{\mathcal{F} \epsilon G(X):(\bar{U})^{0} \epsilon \mathcal{F}\right\}
$$

With the topology determined by the base $\left\{U^{*}: U\right.$ is open in $\left.X\right\}$, the Gleason space $G(X)$ is compact, Hausdorff and extremally disconnected; and if $X$ is a ccc space, then so is $G(X)$.

The cardinality of a set $X$ will be denoted by $|X|$.

We will need the following delta system lemma several times. It is so called because the family $\mathcal{B}$ is a delta system.

Lemma. Let $\mathcal{G}$ be an uncountable family of finite sets. Then there is an uncountable subfamily $\mathcal{B}$ of $\mathcal{G}$ and a fixed set $R$ such that $A \cap B=R$ whenever $A$ and $B$ are distinct members of $B$.

Proof: [8, p. 225]. Since $\mathcal{G}$ is uncountable and the sets are finite, there must be uncountably many members of $\mathcal{G}$ with the same number of elements; therefore we may assume that for some $n,|X|=n$ for all $X$ in $\mathcal{G}$. We proceed by induction on $n$. For $n=1$, we may take $R=\phi$. Assume that the lemma holds for $n=k$ and let $\mathcal{G}$ be an uncountable family of sets each of which has $k+1$ elements. 
If there is some point $a$ which belongs to each set in an uncountable subfamily $\mathcal{C}$ of $\mathcal{G}$, then the induction hypothesis may be applied to the family $\{X \backslash\{a\}$ : $X \in C)$ to yield an uncountable subfamily $B$ of $\mathcal{C}$ and a finite set $R$ such that

$$
(X \backslash\{a\}) \cap(Y \backslash\{a\})=R
$$

for any distinct $X$ and $Y$ in $\mathcal{B}$. Then $X \cap Y=R \cup\{a\}$ for any distinct $X$ and $Y$ in $\mathcal{B}$.

Otherwise, each element $a$ belongs to only countably many members of $\mathcal{G}$, and we construct a disjoint subfamily $\mathcal{B}=\left\{X_{\alpha}: \alpha<\omega_{1}\right\}$ of $\mathcal{G}$ by transfinite induction on $\alpha$, as follows. Assume that we have constructed $X_{\alpha}$ for all $\alpha<\beta$. Then each element of the countable set $U_{\alpha<\beta} X_{\alpha}$ belongs to at most countably many members of $\mathcal{G}$, so there is some $X$ in $\mathcal{G}$ which is disjoint from $U_{\alpha<\beta} X_{\alpha}$. Let $X_{\beta}=X$ and the proof is complete.

\section{Martin's Axiom and products of $c c c$ spaces}

Martin's Axiom [13] states that no compact Hausdorff ccc space is the union of fewer than $2^{\omega}$ nowhere dense sets. Observe that Martin's Axiom (henceforth denoted by $M A$ ) is implied by the Continuum Hypothesis because under $C H, M A$ is an immediate consequence of the Baire category theorem. However, $M A \Rightarrow C H$ because, as is shown in [19], there is a model of $Z F C$ in which $M A$ holds and $\omega_{1}<2{ }^{\omega}$.

In this section we prove that $(M A+\neg C H)$ implies that every product of $c c c$ spaces is a ccc space, and we do it with the help of an interesting theorem about products of $c c c$ spaces (Theorem 2.2) whose proof requires no special axioms.

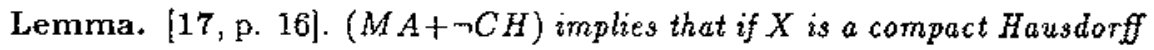
ccc space, then any uncountable family $g=\left\{U_{\alpha}: \alpha<\omega_{1}\right\}$ of nonempty open sets has a cardinality $\omega_{1}$ subfamily with nonempty intersection.

Proof: Consider the set $S$ of all families of disjoint nonempty open subsets of $X$ with the property that if $\mathcal{F} \epsilon S$, then each member of $\mathcal{F}$ meets only countable many members of $G$. If $S$ is empty, we are through. So suppose that $S$ is not empty. Then by Zorn's lemma, $S$ has a maximal member, say $\mathcal{F}$. Since $X$ is a ccc space, $\mathcal{F}$ is countable. Hence there is a member $V$ of $G$ which does not meet any member of $\mathcal{F}$. Then, because $\mathcal{F}$ is maximal, any open subset of $\vec{V}$ must intersect $\omega_{1}$ members of $G$. For each $\beta<\omega_{1}$, define

$$
H_{\beta}=\bar{V} \backslash\left(U_{\alpha}>\beta U_{\alpha}\right) \text {. }
$$

Then $H_{\beta}$ is nowhere dense in the compact Hausdorff $c c c$ space $\vec{V}$. ( $\vec{V}$ is $c c c$ because $V$ is.) Hence $(M A+\neg C H)$ implies that $\bar{V} \neq \cup_{\beta<\omega_{1}} H_{\beta}$. Thus there is $v$ in $\bar{V}$ such that $v \notin \mathrm{U}_{\beta<\omega_{1}} H_{\beta}$, and therefore $v$ belongs to $\omega_{1} U_{\alpha}$ 's.

The following theorem (more generally, Corollary 2.4) was proved independently by K. Kunen, F. Rowbottom, and R.M. Solovay. (See [5, p. 34].) The basic ideas in our proof are from [17, p. 17]. 
Theorem 2.1. $(M A+\neg C H)$ implies that if $X$ and $Y$ are ccc spaces, then $X \times Y$ is a ccc space.

Proof: Suppose that $\left\{U_{\alpha}: \alpha<\omega_{1}\right\}$ is an uncountable family of nonempty open subsets of $X \times Y$. We will show that there are two members of this family which intersect. By shrinking if neccessary, we may assume that each $U_{\alpha}$ is basic. Then there are open sets $V_{\alpha} \subset X$ and $W_{\alpha} \subset Y$ such that $U_{\alpha}=V_{\alpha} \times W_{\alpha}$. The Gleason spaces, $G(X)$ and $G(Y)$, of $X$ and $Y$ are compact, Hausdorff and $c c c$. Hence by the lemma above, there is an uncountable subset $D$ of $\omega_{1}$ such that

$$
\bigcap_{\alpha \epsilon D} V_{\alpha}^{*} \neq \phi \text { and } \cap_{\alpha \epsilon D} W_{\alpha}^{*} \neq \phi .
$$

(Recall from Section 1 that $U^{*}$ is the set of all ultrafilters which contain $(\bar{U})^{0}$.) Let $\beta, \gamma \in D$ with $\beta \neq \gamma$. Then

$$
\left(\overline{V_{\beta}}\right)^{0} \cap\left({ \overline { V _ { \gamma } } } ^ { 0 } \neq \phi \text { and } ( \overline { W _ { \beta } } ) ^ { 0 } \cap \left({\overline{W_{\gamma}}}^{0} \neq \phi .\right.\right.
$$

Hence $V_{\beta} \cap V_{\gamma} \neq \phi$ and $W_{\beta} \cap W_{\gamma} \neq \phi$, and it follows easily that $U_{\beta} \cap U_{\gamma} \neq \phi$.

The $c c c$ has the interesting property that if the product of any two $c c c$ spaces is a $c c c$ space, then the product of any number of $c c c$ spaces is a $c c c$ space. To see this, assume that $c c c$ is preserved by products of two spaces. Then by induction, it is preserved by products with a finite numbers of factors. Then by the following theorem (which seems to have originated in $[14]$ ), it is preserved by arbitrary products.

Theorem 2.2. Suppose that $\left\{X_{i}: i \in I\right\}$ is a family of topological spaces such that $\Pi_{i \in J} X_{i}$ is a ccc space for every finite $J \subset I$. Then $\Pi_{i \in I} X_{i}$ is a ccc space.

Proof: [10, p. 51]. Let $X=\Pi_{i \epsilon I} X_{i}$ and suppose that there exists an uncountable family $\left\{U_{\alpha}: \alpha<\omega_{1}\right\}$ of disjoint nonempty open subsets of $X$. As before, we may assume that each $U_{\alpha}$ is basic. Then by definition of the product topology, for each $\alpha<\omega$, there is a finite subset $F_{\alpha}$ of $I$ such that $U_{\alpha}=\cap\left\{P_{i}^{-1}\left(V_{i}\right): i \in F_{\alpha}\right\}$, where $P_{i}$ is the projection of $X$ onto $X_{i}$, and $V_{i}$ is open in $X_{i}$. By the delta system lemma of Section 1, there is an uncountable subset $D$ of $\omega_{1}$ and a set $R$ such that $F_{\alpha} \cap F_{\beta}=R$ whenever $\alpha, \beta \epsilon D$ and $F_{\alpha} \neq F_{\beta}$. Note that $R$ cannot be empty because $F_{\alpha} \cap F_{\beta}=\phi$ implies $U_{\alpha} \cap U_{\beta}=\phi$. For each $\alpha$ in $D$, let $P\left(U_{\alpha}\right)=\prod_{i \epsilon R} P_{i}\left(U_{\alpha}\right)$. It is not hard to verify that $\left\{P\left(U_{\alpha}\right): \alpha \epsilon D\right\}$ is an uncountable family of disjoint nonempty open subsets of $\Pi_{i \in R} X_{i}$, which is a contradiction.

Since a finite product of separable spaces is separable, the following corollary is an immediate consequence of Theorem 2.2 .

Corollary 2.3. If $\left\{X_{i}: i \in I\right\}$ is a family of separable spaces, then $\Pi_{i \in I} X_{i}$ is a ccc space. 
Corollary 2.4. $(M A+\neg C H)$ implies that if $\left\{X_{i}: i \epsilon I\right\}$ is a family of ccc spaces, then $\Pi_{i \in I} X_{i}$ is a ccc space.

Other consequences of $(M A+\neg C H)$, not all topological, may be found in [4] and [18].

\section{The Laver-Galvin example}

In this section we describe the example due to $R$. Laver and F. Galvin [5], which assumes $C H$, of $c c c$ spaces $X_{0}$ and $X_{1}$ such that $X_{0} \times X_{1}$ is not a $c c c$ space. This will lead (via Gleason spaces) to a compact Hausdorff extremally disconnected $c c c$ space $X$ whose square is not a $c c c$ space. The material in this section is adapted from [2] and [5].

The following lemma can be proved by induction, $A$ proof of a somewhat more general result may be found in $[2$, p. 190].

Lemma 3.1. Let $A$ be a set and for each $n<\omega$, let $\left\{F_{i, n}: i \in I_{n}\right\}$ be a family of disjoint finite subsets of $A$ with $\left|I_{n}\right|=\omega$. Then there are two subsets $A_{0}$ and $A_{1}$ of $A$ such that

$$
\left|\left\{i \in I_{n}: F_{i, n} \subset A_{t}\right\}\right|=\omega
$$

for all $n<\omega$ and $t=0,1$.

Lemma 3.2. Assume that $w_{1}=2^{\omega}$. Then there are two families $\left\{K_{0}(\alpha)\right.$ : $\left.\alpha<\omega_{1}\right\},\left\{K_{1}(\alpha): \alpha<\omega_{1}\right\}$ of subsets of $\omega_{1}$ such that

(i) $K_{0}(\alpha) \cup K_{1}(\alpha)=\alpha$ for all $\alpha<\omega_{1}$, and

(ii) If $\mathcal{F}=\left\{F_{i}: i<\omega\right\}$ is a countably infinite family of disjoint finite subsets of $\omega_{1}$, then there is an ordinal $\lambda$ (depending on $\mathcal{F}$ ) with $\lambda<\omega_{1}$, such that if $\lambda<\alpha<\omega_{1}, \cup \mathcal{F} \subset \alpha, X$ is a finite subset of $\alpha, t \in\{0,1\}$ and

$$
\left|\left\{i<\omega: F_{i} \subset \beta \backslash K_{t}(\beta) \forall \beta \epsilon X\right\}\right|=\omega,
$$

then

$$
\mid\left\{i<\omega: F_{i} \subset \beta \backslash K_{t}(\beta) \forall \beta \in X \cup\{\alpha\} \mid=\omega .\right.
$$

Proof: We begin by computing, under $C H$, the cardinality of the set $S$ of all countably infinite families of disjoint finite subsets of $\omega_{1}$. Clearly $|S| \geq \omega_{1}$ since for each ordinal $\alpha$ such that $\omega \leq \alpha<\omega_{1}$, the family of $\alpha$ singletons is countably infinite and disjoint. To see that $|S| \leq \omega_{1}$, first note that the set of all finite subsets of $\omega_{1}$ has cardinality

$$
\omega_{1}+\left(\omega_{1}\right)^{2}+\left(\omega_{1}\right)^{3}+\cdots=\omega_{1}+\omega_{1}+\omega_{1}+\cdots=\omega_{1} \omega=\omega_{1} .
$$

Hence

$$
|S| \leq \omega_{1}^{\omega}=\left(2^{\omega}\right)^{\omega}=2^{\omega^{2}}=2^{\omega}=\omega_{1} .
$$


Thus $|S|=\omega_{1}$. Let $\left\{\mathcal{F}_{\lambda}: \lambda<\omega_{1}\right\}$ be a well-ordering of $S$ in such a way that for each $\lambda<\omega$, the members of $\mathcal{F}_{\lambda}$ are subsets of $\omega$.

In what follows, $F_{i, \lambda}$ denotes the ith member of the family $\mathcal{F}_{\lambda}$.

We now define the sets $K_{0}(\alpha), K_{1}(\alpha)$ by recursion. We set $K_{t}(\alpha)=\alpha$ for $t=0,1$ and $\alpha \leq \omega$. Let $\omega<\alpha<\omega_{1}$ and suppose that $K_{t}\left(\alpha^{\prime}\right)$ has been defined for $t=0,1$ and $\alpha^{\prime}<\alpha$. Consider the set of all triples $(t, \lambda, X)$ such that $t \in\{0,1\}, \lambda<\alpha, \cup \mathcal{F}_{\lambda} \subset \alpha, X$ is a finite subset of $\alpha$, and

$$
\left|\left\{i<\omega: F_{i, \lambda} \subset \beta \backslash K_{t}(\beta) \forall \beta \epsilon X\right\}\right|=\omega .
$$

This set of triples is countably infinite because $X$ can be the empty set, and if $\lambda<\omega$ then certainly $\cup \mathcal{F}_{\lambda} \subset \alpha$. Let $\left\{\left(t_{n}, \lambda_{n}, X_{n}\right): n<\omega\right\}$ be an enumeration of this set, and for each $n<\omega$ let

$$
I_{n}=\left\{i<\omega: F_{i, \lambda_{n}} \subset \beta \backslash K_{t_{n}}(\beta) \forall \beta \epsilon X_{n}\right\}
$$

Then $\left|I_{n}\right|=\omega$, hence we may apply Lemma 3.1 with $A$ and $F_{i, n}$ replaced by $\alpha$ and $F_{i, \lambda_{n}}$ respectively; then there are disjoint subsets $L_{0}(\alpha)$ and $L_{1}(\alpha)$ of $\alpha$ such that

$$
\left|\left\{i \epsilon I_{n}: F_{i, \lambda_{n}} \subset L_{t}(\alpha)\right\}\right|=\omega
$$

for $n<\omega$ and $t=0,1$. We set

$$
K_{t}(\alpha)=\alpha \backslash L_{t}(\alpha) \text { for } t=0,1
$$

Let us verify that properties (i) and (ii) are satisfied.

(i) Clearly $K_{0}(\alpha) \cup K_{1}(\alpha)=\alpha$ because $L_{0}(\alpha)$ and $L_{1}(\alpha)$ are disjoint subsets of $\alpha$.

(ii) Let $\mathcal{F}=\left\{F_{i}: i<\omega\right\}$ be a countably infinite family of disjoint finite subsets of $\omega_{1}$. Then $\mathcal{F}=\mathcal{F}_{\lambda}$ for some $\lambda<\omega_{1}$. Suppose that $\lambda<\alpha<\omega_{1}$, $\cup \mathcal{F}_{\lambda} \subset \alpha, X$ is a finite subset of $\alpha, t \in\{0,1\}$, and

$$
\left|\left\{i<\omega: F_{i, \lambda} \subset \beta \backslash K_{t}(\beta) \forall \beta \epsilon X\right\}\right|=\omega .
$$

Then $(t, \lambda, X)=\left(t_{n}, \lambda_{n}, X_{n}\right)$ for some $n<\omega$. Hence if we let

$$
J=\left\{i \in I_{n}: F_{i, \lambda} \subset L_{t}(\alpha)\right\},
$$

then $|J|=\omega$. Let

$$
H=\left\{i<\omega: F_{i, \lambda} \subset \beta \backslash K_{t}(\beta) \forall \beta \varepsilon X \cup\{\alpha\}\right\} .
$$

Our goal is to show that $|H|=\omega$, and we will do this by proving that $J \subset H$. Let $i \epsilon J$. Then $F_{i, \lambda} \subset L_{t}(\alpha)$, hence $F_{i, \lambda} \subset \alpha \backslash K_{t}(\alpha)$. Because $i \in I_{n}$ we have

$$
F_{i, \lambda} \subset \beta \backslash K_{t}(\beta) \forall \beta \epsilon X
$$

Thus

$$
F_{i, \lambda} \subset \beta \backslash K_{t}(\beta) \forall \beta \epsilon X \cup\{\alpha\},
$$

and so $i \epsilon H$. Therefore $J \subset H$ and the proof is complete. 
Theorem 3.3. Assume that $\omega_{1}=2^{\omega}$. Then there are ccc spaces $X_{0}$ and $X_{1}$ such that $X_{0} \times X_{1}$ is not a ccc space.

Proof: For $t \in\{0,1\}$, let $\left\{K_{\imath}(\alpha): \alpha<\omega_{1}\right\}$ be the family of Lemma 3.2 and let $X_{t}$ be the set of all functions on $\omega_{1}$ to $\{0,1\}$. Set

$$
V_{t}(\alpha)=\left\{x \in X_{t}: x \mid K_{t}(\alpha) \equiv 0, x(\alpha)=1\right\} \text { for } \alpha<\omega_{1},
$$

and give $X_{t}$ the topology determined by the subbase $\left\{V_{t}(\alpha): \alpha<\omega_{1}\right\}$. The space $X_{0} \times X_{1}$ is not ccc because the uncountable family

$$
\left\{V_{0}(\alpha) \times V_{1}(\alpha): \alpha<\omega_{1}\right\}
$$

consists of disjoint nonernpty open sets. To see that they are disjoint, suppose that there are $\alpha, \alpha^{\prime}$ with $\alpha<\alpha^{\prime}<\omega_{1}$ and

$$
\left(x_{0}, x_{1}\right) \in\left(V_{0}(\alpha) \times V_{1}(\alpha)\right) \cap\left(V_{0}\left(\alpha^{\prime}\right) \times V_{1}\left(\alpha^{\prime}\right)\right) .
$$

Then since

$$
\alpha \epsilon \alpha^{\prime}=K_{0}\left(\alpha^{\prime}\right) \cup K_{1}\left(\alpha^{\prime}\right) .
$$

$\alpha \in K_{t}\left(\alpha^{\prime}\right)$ for some $t$ in $\{0,1\}$; for this $t$, we have $x_{t}(\alpha)=0$ and $x_{t}(\alpha)=1$, a contradiction.

We now verify that $X_{0}$ and $X_{1}$ are $c c c$ spaces. Let $t \epsilon\{0,1\}$ and let $\left\{U_{i}: i<\right.$ $\omega_{1}$ ) be an uncountable family of nonempty basic open subsets of $X_{t}$. For each $i<\omega$ there is a finite subset $G_{i}$ of $\omega_{1}$ such that

$$
U_{i}=\cap_{\alpha \epsilon G_{i}} V_{t}(\alpha)
$$

Then

$$
\left(\mathrm{U}_{\alpha \in G_{i}} K_{t}(\alpha)\right) \cap G_{i}=\phi \text { for all } i<\omega_{1} .
$$

This is so because if we choose $x$ in $U_{i}$, then $x \epsilon V_{t}(\alpha)$ for all $\alpha$ in $G_{i}$, hence $x \mid G_{t} \equiv 1$ and $x \mid \cup_{\alpha \in G_{i}} K_{t}(\alpha) \equiv 0$. Thus the intersection is empty. Applying now the delta system lemma of Section 1 to the family $\left\{G_{i}\right\}$, we may assume without loss of generality that there is a set $R$ such that $G_{i} \cap G_{i^{\prime}}=R$ whenever $i<i^{t}<\omega_{1}$. Let $F_{i}=G_{i} \backslash R$ for each $i<\omega_{1}$. Then if $i<i^{\prime}<\omega_{1}$ and $\gamma \notin K_{t}(\beta)$ for all $\gamma \epsilon F_{i}$ and $\beta \in F_{i^{\prime}}$, it follows that $U_{i} \cap U_{i^{\prime}} \neq \phi$. We outline the verification. First show that

$$
G_{i} \cap\left(\cup_{\alpha \ell} G_{i^{\prime}} K_{t}(\alpha)\right)=\phi
$$

and

$$
G_{\mathbf{i}^{\prime}} \cap\left(\mathrm{U}_{\alpha \in G_{i}} K_{t}(\alpha)\right)=\phi
$$

it then follows that

$$
\left(G_{i} \cup G_{z^{\prime}}\right) \cap\left(\cup_{\alpha \epsilon G_{i} \cup G_{i^{\prime}}} K_{t}(\alpha)\right)=\phi
$$


Now choose $x$ in $X_{t}$ such that $x \mid G_{i} \cup G_{i^{r}} \equiv 1$ and

$$
x \mid \cup_{\alpha \in G_{i} \cup G_{i}} K_{t}(\alpha) \equiv 0
$$

Then $x \in U_{i} \cap U_{i^{\prime}}$. Thus to prove that $\left\{U_{i}: i<\omega_{2}\right\}$ is not a disjoint family, it is enough to show that there are $i, i^{t}$ such that $i<i^{t}<\omega_{1}$ and $\gamma \notin K_{t}(\beta)$ for all $\gamma \epsilon F_{i}$ and $\beta \epsilon F_{i^{\prime}}$. Set $\mathcal{F}=\left\{F_{i}: i<\omega\right\}$ and let $\lambda<\omega_{1}$ be an ordinal satisfying condition (ii) of Lemma 3.2 for $\mathcal{F}$. We may assume that $U \mathcal{F} \subset \lambda$. Because $\lambda+1$ is countable and $\left\{F_{i}: \omega<i<\omega_{1}\right\}$ is uncountable, there is an ordinal $i^{\prime}$ such that $\omega<i^{\prime}<\omega_{1}$ and $\lambda<\alpha$ for all $\alpha$ in $F_{i^{\prime}}$. Then $\cup \mathcal{F} \subset \alpha$ for all $\alpha$ in $F_{i^{\prime}}$. If $F_{\mathrm{i}^{\prime}}=\phi$, then for any $i<i^{\prime}$ we have that $\gamma \notin K_{t}(\beta)$ for all $\gamma \epsilon F_{i}$ and $\beta \epsilon F_{\mathrm{i}^{\prime}}$. Therefore we assume that $F_{i^{\prime}} \neq \phi$ and we let $\left\{\alpha_{1}, \alpha_{2}, \ldots, \alpha_{n}\right\}$ be the elements of $F_{i^{\prime}}$ in the order inherited from $\omega_{1}$. We note that

$$
\left|\left\{i<\omega: F_{i} \subset \beta \backslash K_{t}(\beta) \forall \beta \epsilon \phi\right\}\right|=\omega
$$

and we apply condition (ii) of Lemma 3.2 successively $n$ times with

$$
\alpha=\alpha_{1}, \alpha_{2}, \ldots, \alpha_{n}
$$

and

$$
X=\phi,\left\{\alpha_{1}\right\},\left\{\alpha_{1}, \alpha_{2}\right\}, \ldots,\left\{\alpha_{1}, \alpha_{2}, \ldots, \alpha_{n-1}\right\}
$$

correspondingly. Hence

$$
\left|\left\{i<\omega: F_{i} \subset \beta \backslash K_{t}(\beta) \forall \beta \epsilon F_{i^{+}}\right\}\right|=\omega .
$$

In particular, there is $i<\omega$ such that $\gamma \notin K_{t}(\beta)$ for all $\gamma \epsilon F_{i}$ and $\beta \epsilon F_{i^{\prime}}$. Thus $U_{i} \cap U_{i^{\prime}} \neq \phi$ and so $X_{t}$ is a $c c c$ space.

Corollary. Assuming $C H$, there is a compact Hausdorff extremally disconnected ccc space $X$ such that $X \times X$ is not a ccc space.

Proof: Let $X_{0}$ and $X_{1}$ be the $c c c$ spaces of the above theorem and let $X$ be the disjoint union of the Gleason spaces $G\left(X_{0}\right)$ and $G\left(X_{1}\right)$. Then $X$ is compact, Hausdorff, extremally disconnected and $c c c$ because both $G\left(X_{0}\right)$ and $G\left(X_{1}\right)$ are. To prove that $X \times X$ is not a $c c c$ space, it is enough to show that $G\left(X_{0}\right) \times G\left(X_{3}\right)$ is not a $c c c$ space because $G\left(X_{0}\right) \times G\left(X_{1}\right)$ is homeomorphic to an open (and closed) subset of $X \times X$. Let $\left\{U_{i} \times V_{i}: i<\omega_{1}\right\}$ be an uncountable family of disjoint nonempty basic open subsets of $X_{0} \times X_{1}$. Then, as is not too difficult to verify, $\left\{U_{i}^{*} \times V_{i}^{*}: i<\omega_{1}\right\}$ is an uncountable family of disjoint nonempty open subsets of $G\left(X_{0}\right) \times G\left(X_{1}\right)$. Thus $G\left(X_{0}\right) \times G\left(X_{1}\right)$ is not a $c c c$ space.

Before leaving this section we remark that S. Argyros, S. Mercourakis and S. Negrepontis have proved the existence, under $C H$, of a Corson-compact $c c c$ space whose square is not $c c c[1]$. 


\section{The Souslin problem}

Suppose that $X$ is a totally ordered set satisfying

(a) $X$ has no first or last element,

(b) $X$ is connected in the order topology, and

(c) $X$ is separable in the order topology.

Then $X$ is linearly isomorphic to $R$. (A proof is in [3, p.8].) In 1920, the Russian mathematician $M$. Souslin [20] asked whether (c) can be replaced by

(c') $X$ is ccc in the order topology.

A positive answer to Souslin's question has come to be known as Souslin's $H y_{t}$ othesis $(S H)$. The axion $S H$ is undecidable in $Z F C$ and is implied by $(M A+\neg C H)([7],[21],[19])$. Indeed, to quote D. Fremlin [4, p. 184], Souslin's problem was the original stimulus for the invention of Martin's axiom.

A Souslin line is a totally ordered set satisfying properties (a), (b) and (c'), but not (c). Thus the existence of a Souslin line is equivalent to the negation of $S H$. G. Kurepa proved [11] that if $X$ is a Souslin line, then $X \times X$ is not a ccc space. (A proof may be found in $[10, p .66]$.) There is also the notion of a Souslin tree; it is shown in [16] that the existence of a Souslin tree is equivalent to the existence of a Souslin line.

\section{Property $(K)$}

A topological space is said to have property (K) (after Knaster [9]) if each uncountable family of open sets contains an uncountable subfamily in which every two sets have nonempty intersection. Clearly every space with property $(K)$ is a $c c c$ space. The converse is true under $(M A+\neg C H)$ (4, Theorem 41 $A]$ and is false under $C H$. To verify the latter, we make use of Marczewski's theorem [12] that a product of spaces has property $(K)$ if (and only if) each space has property $(K)$. Recall from Section 3 that there exists, under $C H$, a ccc space $X$ such that $X \times X$ is not a ccc space. Then $X$ does not have property $(K)$ because otherwise $X \times X$ would have property $(K)$ and thus be a $c c c$ space.

The reader has probably noticed that another approach (in Section 2) to proving that $(M A+\neg C H)$ implies that a product of $c c c$ spaces is a $c c c$ space would be to combine Fremlin's theorem that under $(M A+\neg C H)$ every ccc space has property $(K)$, with Marczewski's theorem quoted above.

For open problems and additional references involving ccc spaces, the reader may consult $[2$, Chapter 7$]$ and $[4, \oint 44]$. 


\section{References}

1. S. ARgYros, S. MERCoURAKIS AND S. NEGREPONTIS, Analytic properties of Corson-compact spaces, General topology and its relations to modern analysis and algebra, Sigma Ser. Pure Math. V (1983), Prague. 3, Heidermann, Berlin.

2. W.W. COMFort AND S. NeGrepontis, "Chain Conditions in Topology," Cambridge university Press, 1982.

3. K.J. DEVLIN AND H. JoHnSBRATEN, "The Souslin Problem, Lecture Notes in Mathematics," Springer-Verlag, 1974. vol.405

4. D.H. FREMLIN, Consequences of Martin's Axiom, Cambridge University Press (1984).

5. F. GaLVIN, Chain conditions and products, Fund. Math. 108 no.1 (1980), $33-48$.

6. P.R. Halmos, "Naive Set Theory," Van Nostrand, 1960.

7. T. JECH, Non-provability of Souslin's hypothesis, Comment. Math. Univ. Carolinae 8 (1967), 291-305.

8. T. JECH, "Set Theory," Academic Press, 1978.

9. B. KNASTER, Sur une propriété charactéristique de l'ensemble des nombres réels, Rec. Math./Mat. Sbornik] N.S. 16(58) (1945), 281-290.

10. K. Kunen, "Set Theory," North Holland, 1980.

11. G. KUREPA (DJURo KUREPA), Sur une propriété charactéristique du continu linéaire et le problème de Suslin, Acad. Serbe Sci. Publ. Inst. Math. 4 (1952), 97-108.

12. E. MARCZEWSKI, Séparabilité et multiplication cartésienne des espaces topologiques, Fund. Math. 34 (1947), 127-143.

13. D.A. MARTIN AND R.M. SOlovay, Internal Cohen extensions, Ann. Math. Logic 2 (1970), 143-178.

14. N. NOBLE AND $M$. ULMER, Factoring functions on Cartesian products, Trans. Amer. Math. Soc 163 (1972), 329-339.

15. K.A. ROSS AND A. H. STONE, Products of separable spaces, Amer. Math. Monthly 71 (1964), 398-403.

16. M.E. Rudin, Souslin's Conjecture, Amer. Math. Monthly 76 (1969), $1113-1119$.

17. M.E. Rudin, Lectures on Set-Theoretic Topology, American Mathematical Society (1975).

18. J.R. Shoenfield, Martin's axiom, Amer. Math. Monthly 82 (1975), 610-617.

19. R.M. SOlOVAY AND S. Tennenbaum, Iterated Cohen extensions and Souslin's problem, Ann. of Math.(2) 94 (1971), 201-245.

20. M. Souslin, Problème 3, Fund. Math. 1 (1920), 223. 
21. S. Tennenbaum, Souslin's problem, Proc. Nat. Acad. Sci U.S.A. 59 (1968), 60-63.

\author{
Department of Mathematics \\ Rosemont College \\ Rosemont \\ PA 19010. U S A
}

Rebut el 19 d'Octubre de 1988 